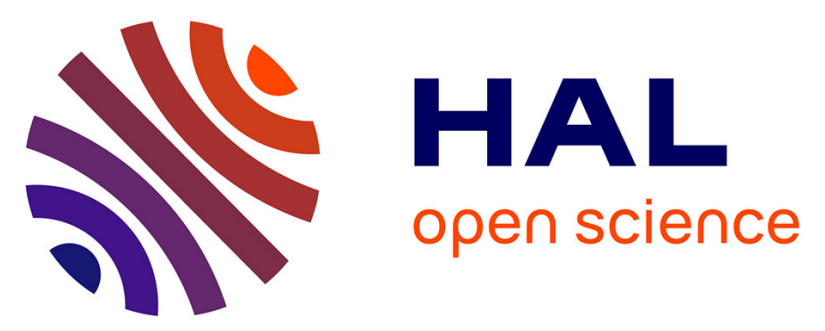

\title{
Kerr nonlinearity compensation in a 5x28-GBd PDM 16-QAM WDM system using fiber-based optical phase conjugation
}

Francesco da Ros, Isaac Sackey, Robert Elschner, Thomas Richter, Christian Meuer, Markus Nölle, Mahmoud Jazayerifar, Klaus Petermann, Christophe Peucheret, Colja Schubert

\section{To cite this version:}

Francesco da Ros, Isaac Sackey, Robert Elschner, Thomas Richter, Christian Meuer, et al.. Kerr nonlinearity compensation in a 5x28-GBd PDM 16-QAM WDM system using fiber-based optical phase conjugation. 40th European Conference on Optical Communication (ECOC 2014), Sep 2014, Cannes, France. paper P.5.3, 10.1109/ECOC.2014.6963897 . hal-01069388

\section{HAL Id: hal-01069388 https://hal.science/hal-01069388}

Submitted on 29 Sep 2014

HAL is a multi-disciplinary open access archive for the deposit and dissemination of scientific research documents, whether they are published or not. The documents may come from teaching and research institutions in France or abroad, or from public or private research centers.
L'archive ouverte pluridisciplinaire HAL, est destinée au dépôt et à la diffusion de documents scientifiques de niveau recherche, publiés ou non, émanant des établissements d'enseignement et de recherche français ou étrangers, des laboratoires publics ou privés. 


\title{
Kerr Nonlinearity Compensation in a 5×28-GBd PDM 16-QAM WDM System Using Fiber-Based Optical Phase Conjugation
}

\author{
F. Da Ros ${ }^{(1,2)}$, I. Sackey ${ }^{(2,3)}$, R. Elschner ${ }^{(2)}$, T. Richter ${ }^{(2)}$, C. Meuer ${ }^{(2,3)}$, M. Nölle ${ }^{(2)}$, M. Jazayerifar ${ }^{(3)}$, \\ K. Petermann ${ }^{(3)}$, C. Peucheret ${ }^{(4)}$, and C. Schubert ${ }^{(2)}$ \\ ${ }^{(1)}$ Department of Photonics Engineering, Technical University of Denmark, DK-2800 Kgs. Lyngby, \\ Denmark (Email: fdro@fotonik.dtu.dk) \\ ${ }^{(2)}$ Fraunhofer Institute for Telecommunications, Heinrich Hertz Institute, Einsteinufer 37, 10587 Berlin \\ (3) Technische Universität Berlin, Fachgebiet Hochfrequenztechnik, Einsteinufer 25, 10587 Berlin, \\ Germany, (Email: isaac.sackey@hhi-extern.fraunhofer.de) \\ (4) FOTON Laboratory, CNRS UMR 6082, ENSSAT, University of Rennes 1, F-22305 Lannion, France
}

Abstract Effective Kerr nonlinearity mitigation is experimentally demonstrated using optical phase conjugation in the middle of an 800-km dispersion-compensated link for a 5-channel WDM 28-GBd PDM 16-QAM signal. A Q-factor improvement of $0.9 \mathrm{~dB}$ over no mitigation allows a $B E R<3.8 \times 10^{-3}$.

\section{Introduction}

To respond to the ever increasing demand for capacity, current optical networks are migrating towards higher bitrates by the use of more complex modulation formats, thus increasing the required optical signal-to-noise ratio (OSNR) for error-free transmission. In order to accommodate for such requirements, higher optical power levels need to be launched into the transmission link. However, nonlinear distortion caused by the Kerr effect in optical fibers sets an upper bound on the total power that can be transmitted before degrading the signal performance ${ }^{1}$. Therefore, being able to compensate for nonlinear distortion would allow increasing the received optical OSNR without compromising the transmission reach.

Along such direction, several techniques have been proposed, either in the electrical or in the optical domain. Digital back-propagation algorithms are being investigated to invert the Kerr effect using digital signal processing ${ }^{2}$. However, this approach scales poorly with the number of channels due to the high requirements in terms of electrical receiver bandwidth and computational power.

Alternatively, the compensation can be achieved optically using optical phase conjugation (OPC) to provide mid-span spectral inversion (MSSI). With the OPC placed in the middle of the link, the spectral inversion allows for compensating the nonlinear distortion caused by the first half of the link through propagation in the second half ${ }^{3,4}$.

Recently, a hybrid approach making use of a coherent superposition between a signal and its phase-conjugated copy propagating through the same link has also been demonstrated ${ }^{5}$. This technique, however, has the main drawback of halving the spectral efficiency as two copies of the same signal need to be transmitted.
In this work, we experimentally evaluate the benefit of the MSSI approach using optical phase conjugation in order to provide nonlinearity compensation for a 5-channel wavelength-division multiplexed (WDM) 28-GBd polarization-division multiplexed (PDM) 16-quadrature amplitude modulation (QAM) signal. Compared to Ref. 4 we demonstrate polarization insensitive operation and minimize the penalty introduced by the OPC device by improving the power transparency and using a dual-pump scheme ${ }^{6}$. The Q-factor improvement provided by the compensation is investigated for different dispersion-compensated transmission lengths with clear improvement reported for all the lengths considered. The OPC operation allows reaching a target bit-error ratio (BER) below $3.8 \times 10^{-3}$, i.e. the hard-detection forward error correction (HD-FEC) threshold, for 800-km transmission, while all channels had a BER>HD-FEC threshold without compensation.

\section{Experimental setup}

The experimental setup is sketched in Fig. 1. It consists mainly of a 28-GBd PDM 16-QAM transmitter, a dispersion-compensated optical transmission link with a dual-pump polarization insensitive fiber optical parametric amplifier (FOPA) used to provide OPC in the middle of the link, and a coherent receiver followed by off-line digital signal processing.

The signal is generated by five external-cavity lasers (ECLs) on a $50-\mathrm{GHz}$ grid centered at 193.40 THz and modulated in the 16-QAM format using an IQ modulator driven by two-channels of a 56-GSamples/s arbitrary waveform generator (AWG). After a split-anddelay PDM emulator (PolMux) implementing a delay of more than 500 symbols between the two polarizations, an interleaving stage based on a wavelength selective switch (WSS) fully decorrelates all five channels with a minimum 


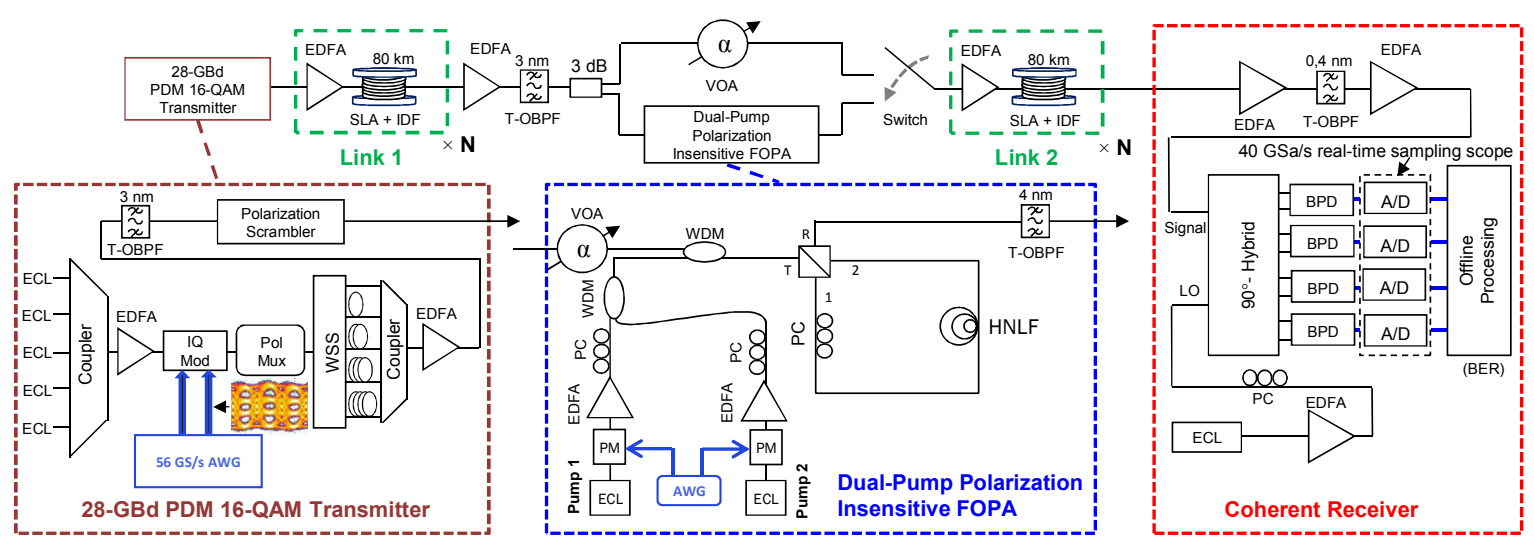

Fig. 1: Experimental setup.

inter-channel delay of 100 symbols in order to mimic a real-world scenario. The $1.12 \mathrm{~Tb} / \mathrm{s}$ signal is then amplified by an erbium-doped fiber amplifier (EDFA), out-of-band amplified spontaneous emission (ASE) noise is filtered out by a tunable optical bandpass filter (T-OBPF) with a 3-nm full-width half-maximum (FWHM) bandwidth and a fast scrambler is used to randomize the state-of-polarization and thus demonstrate polarization-insensitive operation.

Each of the $2 \times \mathrm{N}$ dispersion-compensated spans constituting the transmission link consists of an EDFA and a combination of super large area (SLA) fiber followed by inverse dispersion fiber (IDF) for a total span length of $80 \mathrm{~km}$. After the first $\mathrm{N}$ spans (Link 1), the signal is amplified by an EDFA followed by a 3-nm wide T-OBPF to suppress ASE noise, and then split into two paths via a 3-dB coupler: one goes through the FOPA, the other bypasses it, skipping the OPC.

The FOPA performing the OPC operation consists of a dual-pump polarization-insensitive scheme based on a polarization diversity loop ${ }^{6,7}$.

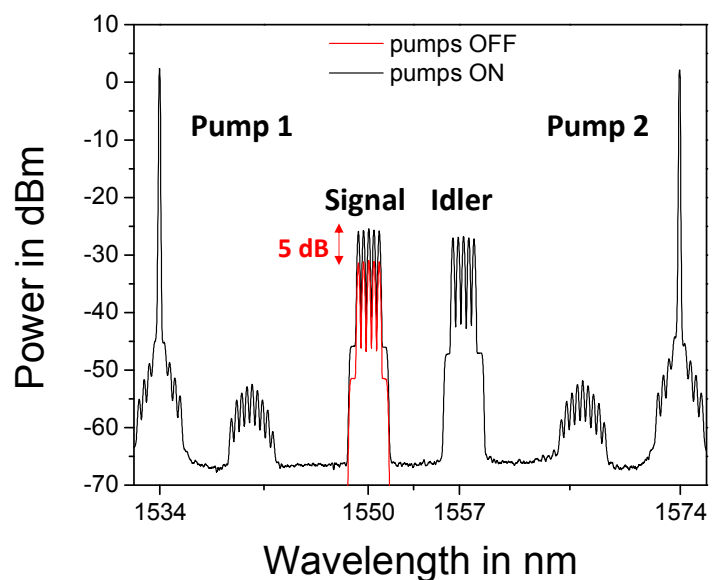

Fig. 2: Spectra ( $0.1 \mathrm{~nm}$ resolution) at the output of the HNLF for pumps ON and pumps OFF conditions with the 5-dB ON-OFF gain clearly shown.

The signal is coupled together with two continuous wave (CW) pumps at $1534 \mathrm{~nm}$ and $1574 \mathrm{~nm}$ through a WDM coupler. The pumps are phase modulated by two frequency tones at $69 \mathrm{MHz}$ and $253 \mathrm{MHz}$ in counterphasing operation to increase the stimulated Brillouin scattering (SBS) threshold while minimizing the degradation on the idler due to pump phase noise transfer ${ }^{7}$. Signal and pumps are then injected into the highly nonlinear fiber (HNLF) through a polarization beam splitter (PBS). Two polarization controllers (PCs) are used to ensure the pumps power levels are the same in the clock wise and counter-clock wise propagation directions, thereby providing similar gain to both polarization components of the signal, ${ }^{6,7}$. The polarization dependent gain (PDG) was optimized below $0.3 \mathrm{~dB}$ over the bandwidth of interest by temporarily switching off the PDM emulator. The HNLF length, zero dispersion wavelength, dispersion slope, nonlinear coefficient and attenuation are $300 \mathrm{~m}$, $1555.6 \mathrm{~nm}, 0.019 \mathrm{ps} /\left(\mathrm{nm}^{2} \cdot \mathrm{km}\right), 16.3 \mathrm{~W} / \mathrm{km}$ and $1.4 \mathrm{~dB} / \mathrm{km}$, respectively. The total signal power into the diversity loop is optimized to $2 \mathrm{dBm}$ in order to avoid operating the FOPA in saturation ${ }^{8}$ but at the same time keeping the OSNR degradation low ${ }^{6}$. A total pump power of $29.2 \mathrm{dBm}$ provides an ON-OFF gain of $5 \mathrm{~dB}$ as shown in Fig. 2, to minimize the penalty introduced by the OPC operation ${ }^{6}$. A T-OBPF (4-nm FWHM bandwidth) at the output of the PBS selects the phase-conjugated idler, suppressing signal and pumps.

A switch at the input of the following $\mathrm{N}$ spans (Link 2) allows selecting either the original signal having propagated over Link 1 (without OPC) or the idler generated in the FOPA (with OPC). Please note that a variable optical attenuator (VOA) in the path without OPC ensures the same input power is injected to the first EDFA of Link 2 in the two scenarios, thereby ensuring the same noise accumulation behavior.

At the output of the transmission link the channels are amplified by the receiver's pre-amplifier. The channel under investigation is selected by a narrow-band T-OBPF (0.4-nm FWHM bandwidth), amplified and combined with a $100-\mathrm{kHz}$ local oscillator (LO) in a $90^{\circ}$ optical hybrid. Four balanced photodiodes (BPD) at the 
hybrid outputs are connected to four $20-\mathrm{GHz}$ channels of a real-time sampling scope providing the analog-to-digital (A/D) conversion. Finally offline processing is performed including resampling, frequency-offset compensation, blind adaptive time-domain equalization using constant- and multi-modulus algorithms, carrier-phase estimation by blind phase search, demapping and error counting.

\section{Results and discussion}

In order to investigate the effectiveness of the nonlinearity compensation in the OPC stage, the $Q$-factor, derived from the BER averaged over the five WDM channels, has been evaluated by varying the power launched into each span.

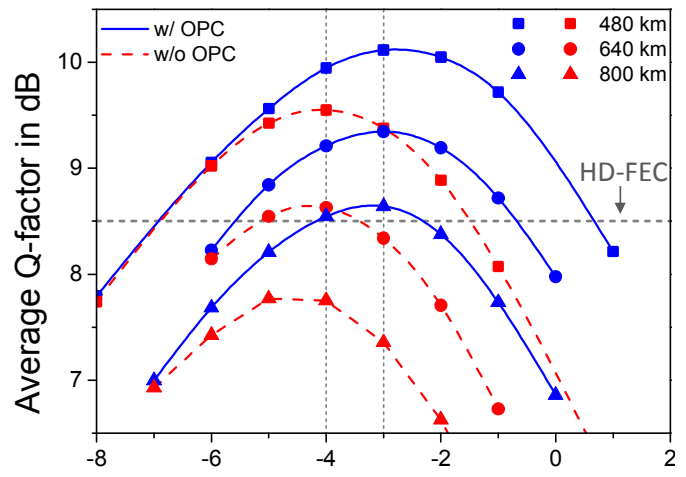

Launched power in $\mathrm{dBm} / \mathrm{ch} / \mathrm{pol}$

Fig. 3: Averaged $Q$-factor after transmission for increasing link lengths as a function of the launched power per channel per polarization in each span.

Fig. 3 shows the Q-factor as a function of launched power with and without OPC for three different transmission lengths corresponding to 6,8 and 10 spans ( $N=3,4$ and 5 ). As can be clearly seen, OPC does not reduce the performance of the system in the linear regime for all the transmission lengths considered. In the nonlinear regime, the nonlinearity

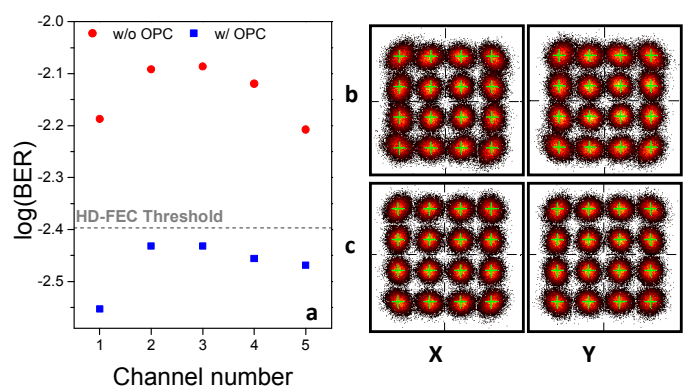

Fig. 4: (a) BER after $800 \mathrm{~km}$ transmission for the five channels at the optimum launched power without $(-4 \mathrm{dBm})$ and with $(-3 \mathrm{dBm})$ OPC. Constellation diagrams for the best channel without OPC $(\mathrm{CH} 5, \mathrm{~b})$ and the worst with OPC $(\mathrm{CH} 2, \mathrm{c})$. Green crosses show the position of the clusters for a reference constellation.

compensation provided by OPC enables improvements of the Q-factor of $0.56 \mathrm{~dB}$, $0.71 \mathrm{~dB}$ and $0.9 \mathrm{~dB}$, for $480 \mathrm{~km}, 640 \mathrm{~km}$ and $800 \mathrm{~km}$, respectively, provided only $1 \mathrm{~dB}$ higher power per channel is launched in the spans.
Interestingly, some improvement is achieved even when the launched power is equal to its optimum value without OPC $(-4 \mathrm{dBm}$ per channel per polarization).

Furthermore, Fig. 3 shows that for $800 \mathrm{~km}$ transmission without OPC, the nonlinear degradation is so severe that the HD-FEC threshold $\left(\mathrm{BER}=3.8 \times 10^{-3}\right.$ ) is not reached. In contrast, employing the OPC in the middle of the link, all the channels show a BER below the HD-FEC threshold, as summarized in Fig. 4(a). Note that the wavelength-dependent BER is related to the number of neighbors of each channel, inner channels being more subjected to nonlinear distortion than outer ones.

After transmission, the power-dependent nonlinear phase rotation causes the outer clusters of the constellation to be rotated with respect to the four inner clusters (Fig. 4(b)). However, the use of OPC mitigates the effect, as shown in Fig. 4(c).

\section{Conclusions}

We have demonstrated effective Kerr nonlinearity compensation through a polarization-insensitive OPC scheme for a $5 \times 28-G B d$ PDM 16-QAM signal at a total gross bitrate of $1.12 \mathrm{~Tb} / \mathrm{s}$. A Q-factor improvement up to $0.9 \mathrm{~dB}$ is reported, enabling receiving the signal after $800 \mathrm{~km}$ dispersion-compensated transmission with a BER below the HD-FEC threshold while all channels had a BER>HD-FEC threshold without compensation.

\section{Acknowledgements}

This work was funded by the Deutsche Forschungsgemeinschaft (DFG) (grants GR 3774/1-1 and PE 319/26-1) and the Danish Research Council for Technology and Production Sciences (project 09-066562).

\section{References}

[1] A. D. Ellis, et al., "Approaching the non-linear Shannon limit," JLT, Vol. 28, p. 423 (2010).

[2] D. Rafique, et al., "Digital back-propagation for spectrally efficient WDM 112 Gbit/s PM m-ary QAM transmission", Opt. Express, Vol. 19, p. 5219 (2011).

[3] H. Hu, et al., "Fiber nonlinearity compensation of an 8channel WDM PDM-QPSK signal using multiple phase conjugations", Proc. OFC 2014, paper M3C.2.

[4] L. B. Du, et al, "Fiber nonlinearity compensation for OFDM super-channels using optical phase conjugation", Opt. Express, Vol 20, p. 19921 (2012).

[5] X. Liu, et al., "Phase-conjugated twin waves for communication beyond the Kerr nonlinearity limit", Nat. Photon, Vol. 7, p. 560 (2013).

[6] I. Sackey, et al., "Design and performance evaluation of an OPC device using a dual pump polarizationindependent FOPA", ECOC 2014, submitted.

[7] T. Richter et al., "Parametric amplification and wavelength conversion of single and dual polarization DQPSK signals," IEEE JSTQE, Vol. 18, p. 988 (2012).

[8] F. Da Ros, et al., "Impact of Gain Saturation on the Parametric Amplification of 16-QAM Signals", Proc. ECOC 2013, paper We.2.A.3. 